\title{
Frontal Gamma Asymmetry in Response to Soft Skills Stimuli: A Pilot Study
}

\author{
Ronald J. Bonnstetter ${ }^{1,2,3^{*}}$, Dustin Hebets ${ }^{1,2}$, and Nancy L. Wigton ${ }^{4,5}$ \\ ${ }^{1}$ Center for Applied Cognitive Research, Scottsdale, Arizona, USA \\ ${ }^{2}$ Target Training International, Ltd., Scottsdale, Arizona, USA \\ ${ }^{3}$ University of Nebraska - Lincoln, Lincoln, Nebraska, USA \\ ${ }^{4}$ Grand Canyon University, Phoenix, Arizona, USA \\ ${ }^{5}$ Applied Neurotherapy Center, LLC, Scottsdale, Arizona, USA
}

\begin{abstract}
Objective: The purpose of this pilot study was to explore associations between self-reported rank ordering of a set of 23 job-related soft skills and frontal gamma (38 to $42 \mathrm{~Hz}$ ) asymmetry emerging during exposure to the same set of soft skills. Method: Fourteen individuals responded to a soft skill assessment, then were exposed to a randomized list of the same soft skills' key words while collecting electroencephalographic (EEG) data, using a new implementation of standardized low-resolution brain electromagnetic tomography (sLORETA) to analyze and view voxel images of real-time brain activity. A differential calculation, as a measure of approach or avoidance to the key word stimulus (Approach-Avoidance-Differential; AAD), was used to quantify the asymmetry in response to the stimuli. Spearman's Rank correlations $\left(r_{s}\right)$ were calculated for the paired occurrences between the selfreported ranking of the soft skills and the AAD. Results: Overall, $71 \%$ of the cases resulted in correlations, indicating soft skill directionality response. Reduction in gamma response intensity was seen when participants' ranked their highest to lowest soft skills, as indicated in their self-reported assessments. Conclusions: These results will inform further organizational neuroscience research which has potential to lead to a new approach to self-report validation and methods to detect individual approach or avoidance biases which impact self-reporting assessments.
\end{abstract}

Keywords: gamma asymmetry, soft skills, precognition, industrial/organizational psychology, organizational neuroscience, sLORETA, approach-avoidance

Citation: Bonnstetter, R. J., Hebets, D., \& Wigton, N. L. (2015). Frontal Gamma Asymmetry in Response to Soft Skills Stimuli: A Pilot Study. NeuroRegulation, 2(2), 70-85. http://dx.doi.org/10.15540/nr.2.2.70

*Address correspondence to: Ronald J. Bonnstetter, PhD, Center for Applied Cognitive Research, 17785 North Pacesetter Way, Scottsdale, AZ 85255, USA. Email: ron@ttiltd.com

Copyright: () 2015. Bonnstetter et al. This is an Open Access article distributed under the terms of the Creative Commons Attribution License (CC-BY).
Edited by:

Barbara Hammer, PhD, Private Practice, Clinical Psychology and Neurofeedback, California, USA

\section{Reviewed by:}

Rex Cannon, PhD, Positive Brain Training, Florida, USA Barbara Hammer, PhD, Private Practice, Clinical Psychology and Neurofeedback, California, USA

\section{Introduction}

The concept of self-report is a key element in many phases of psychology, counseling, as well as industrial-organizational coaching and human resource management. It is often the only means to probe the internal world of a person. However, selfreports are limited by key issues, including the level of awareness, honesty, and ability to express internal thoughts and feelings, which is highly variable across individuals (Kanai \& Rees, 2011). Self-reporting assessments, frequently in the form of ipsative or force choice assessments, are therefore suspect in the absence of crossverification. Objective measures, including physiological responses, nonverbal expressions, and other observables, can be considered to supplement self-reports.

A further limitation of ipsative assessments, as outlined in Bedwell, Fiore, and Salas (2011, 2014), are that several particular biases can influence selfreport measures: Consistency motif, social desirability, acquiescence biases, and self-serving biases. While a comprehensive discussion of these influences is beyond the scope of this paper, a brief 
overview will add clarity to this aspect of self-report assessments. The consistency motif implies that individuals may attempt to create consistency in their thoughts and feelings and, as a result, maintain consistency in their responses rather than dealing with each question individually. Social desirability addresses the need for social approval and acceptance that can lead to behaviors deemed culturally acceptable, thus presenting themselves in a favorable manner, regardless of their true feelings or tendencies. Acquiescence bias occurs when respondents generally agree (or disagree) with questionnaires, regardless of content. This may result in some components of an assessment seeming to be related, when in fact, they are not. Self-serving bias may occur when people attribute the more positive aspects of their performance to their own traits or dispositions and poor performance to external factors. This bias may result in higher self-ratings on assessment questions regarding mastery levels. Thus, it is crucial that participants be truthful to themselves and that the assessment output is only as accurate as the input. To address these concerns, assessments many times use checks of internal consistency, validity and reliability, and norm comparisons. Ultimately, however, a brain-based measure would be optimal, if it could provide a meaningful indicator of emotional, cognitive, and motivational states and serve as correlation to self-reported responses.

To begin investigating the potential for such a brainbased measure, we hypothesized that a soft skill assessment tool could be administered and then correlated to real-time brain activity. The concept leading to these soft skills, also referred to as competencies, were first described by McClelland (1961); thus, leading to the present-day competency-based job descriptions commonly used in the field of industrial-organizational psychology.

Building on this workplace skill concept, cognitive research has begun to illuminate how intrapersonal and interpersonal competencies are crucial to daily interactions (e.g., National Research Council, 2008, 2012). When matching a person to a job, it becomes crucial that we be able to predict not just what a person knows, but rather, what they are capable of knowing. Furthermore, research has shown that these competencies are not fixed but are developed by prior experience (Yeager \& Walton, 2011), and thus should be tied to memories and emotions associated with those experiences; and therefore, should be traceable in brain imaging. The concept of capacity to know becomes paramount as capacity implies that something needs to be in place for learning to occur. The work of Dweck (2006) refers to potential barriers to learning as a "fixed mindset." A fix mindset is a belief about one's ability that can block learning; and many times are associated with past negative events. It is for this reason that we must separate what a person knows from what they are capable of knowing. Simply providing the learning opportunity without first exposing hidden fix mindsets may not result in learning. It is these negative memories and corresponding emotions that many times must be addressed before learning can occur.

The National Research Council's 2012 report of 21st century skills challenges us to recognize the critical role soft skills play in our lives, both on and off the job. They stress the need for students and working adults to be able to identify and develop skills that include innovation, creativity, problem solving, critical thinking, communication, collaboration, and self-management; all of which are among the jobrelated competencies assessed in this study. In other words, the challenge has been to develop valid and accurate assessment instruments that allow an individual, or an employer, the ability to assess these soft skills quickly and with confidence.

In the quest to investigate the neural underpinnings of individuals' internal processes, as related to industrial and organizational psychology, an entire field termed organizational neuroscience (also called neuroleadership) has begun to emerge; which can further be conceptualized as a branch of the greater field of social cognitive neuroscience (Lafferty \& Alford, 2010; Rock, 2008). The advent of functional magnetic resonance imaging (fMRI) has greatly advanced these efforts. The fMRI signal is produced when changes in blood oxygenation and flow are detected secondary to neuronal activity; thus, when more oxygen is consumed in response to increased brain activity this response is represented in brain activation maps indicating localization of mental processes (Arthurs \& Boniface, 2002). Its use in cognitive neuroscience began in the 1990s, with its most prevalent contribution being the discovery that small areas of brain function can be associated with the act of mentalizing, together with its spatial resolution at the size of 1 or $2 \mathrm{~mm}$ (Arthurs \& Boniface, 2002; Mitchell, 2008). As an example of this line of research, Tabibnia, Satpute, and Lieberman (2008) were able to compare brain activity and self-reported measures to evaluate perceptions of fairness versus unfairness. However, some research from this field is beginning to incorporate quantitative electroencephalography (qEEG) technology to investigate neural measures, 
or signatures, of internal processes. For example, Waldman, Balthazard, and Peterson (2011) used qEEG to link coherence and inspirational leadership traits, wherein they found a correlation between right frontal coherence and socialized visionary communication. In addition to being far less costly to implement and easier to access, one distinct advantage of qEEGs over fMRIs is improved temporal resolution; meaning that $\mathrm{qEEGs}$ can record neural activity faster, in terms of milliseconds (Hüsing, Jäncke, \& Tag, 2006).

One such qEEG technology is low-resolution brain electromagnetic tomography (LORETA). LORETA incorporates a mathematical inverse solution of surface EEG data, which can provide cortical source localization, and generates three-dimensional images, similar to those produced by fMRI data (Thatcher, 2013). The LORETA algorithm creates an estimate of brain activity, termed current source density (CSD), in a virtual space representing cortical structures, encompassing 2,394 coordinates, expressed as $7 \mathrm{~mm}^{3}$ sized voxels (The KEY Institute for Brain-Mind Research, 2014). A new generation of the algorithm, standardized LORETA (sLORETA; Pascual-Marqui, 2002), advances this concept, and bases the computations on a standardized CSD such that the voxel size is $5 \mathrm{~mm}^{3}$ for a total of 6,239 voxels.

\section{The Role of Approach-Avoidance Asymmetry}

While advancements in EEG imaging, quantitative, and source localization analysis are key to this paper, the underlying concept of frontal lobe asymmetry provides the theory bases and can be traced back to Davidson, Schwartz, Saron, Bennett, and Goleman (1979) where they first described the use of scalp-recorded EEG asymmetry and the possible connection to emotional processes. What followed was a plethora of studies documenting the role of frontal lobe approach-avoidance asymmetry in emotional processing and decision making (Davidson, 1992, 2000, 2002, 2004; Davidson, Ekman, Saron, Senulis, \& Friesen, 1990; Gordon, Barnett, Cooper, Tran, \& Williams, 2008; HarmonJones 2004; Nitschke, Heller, Etienne, \& Miller, 2004; Rock, 2008; Rolls, 1999). Davidson, Pizzagalli, Nitschke, and Kalin (2003) summarized the research up to that point by proposing that greater left-side prefrontal cortex activity appeared to be associated with approach-related and goaldirected action planning, while the right suggests avoidance-related emotions.
While the ability to differentiate approach (reward) from avoid (threat) is in itself noteworthy, it is important to understand the bases of this process is directly tied to emotional expressions. Gordon et al. (2008) defines emotions as "adaptive actions tendencies that are mobilized by signals of potential danger or reward. They involve a 'feedforward' mode of brain and body activity that is triggered automatically and without the need for conscious awareness of the triggering signal" (p. 349). They refer to this response to stimuli as a nonconscious emotional reaction, while Collura, Zalaquett, Bonnstetter, and Chatters (2014) define this limbic system processing prior to cognitive awareness as a precognition. Naccache et al. (2005) explains that the limbic networks can process threat and reward cues within 200ths of a second, thus supplying a continuous nonconscious response to every interaction we have, all day long. Being able to detect the corresponding brain activity, when these precognitions occur, provides a direct link to the emotions and experiences behind our decisions, and exposes our thought processing before conscious thoughts or self-regulation can take place (Gordon et al., 2008).

It is important to note that through the 1990s research examining and documenting the concept of approach-avoidance was confined to slower frequency analysis, primarily alpha asymmetry. However, this began to change with a series of experiments (Pizzagalli, Greischar, \& Davidson, 2003; Pizzagalli, Nitschke, et al., 2002; Pizzagalli, Pascual-Marqui, et al., 2001) which included a focus on frequencies in addition to alpha, such as theta, beta, and to a lesser extent, gamma; wherein frontal asymmetries were at times found (Davidson, 2004). Then, Oakes et al. (2004) correlated LORETA CSD to regional glucose metabolism with positron emission tomography, where they found that while alpha did show an expected asymmetry relation, the frequency band most consistently and strongly associated with glucose metabolism was gamma; with localization primarily in the frontal lobe region. As a result of these studies, Davidson (2004) concluded an important aspect in advancing this line of research would be exploring frequencies other than alpha to garner additional information. This identified gap in the research, combined with the noted aspect of gamma discovered by Oakes et al. (2004), provides the basis for this study with its focus on gamma asymmetry. Moreover, the vast majority of this line of research has implemented surface-recorded EEG data, with an inclusion of LORETA source localization to a lesser degree. Yet, recent advances in qEEG applications have 
demonstrated it may be possible to use a new implementation of sLORETA to both analyze and view voxel images of real-time gamma brain activity that may reflect emotional states as related to precognitive activity (Collura, Bonnstetter, \& Zalaquett, 2014; Collura, Zalaquett, et al., 2014).

Our past research and data in assessing workplace soft skills has shown that past experiences, combined with emotional connections to those experiences, are a key component to soft skill development. This research, coupled with frontal asymmetry literature, leads us to hypothesize that frontal asymmetry would correlate to the emotionally laden component of self-reported soft skill rankings. Therefore, this pilot study sought to detect sLORETA-derived real-time brain activity within this approach-avoidance framework, and focused on the comparison of soft skills assessment and precognitive response gamma (38 to $42 \mathrm{~Hz}$ ) asymmetry. The main purpose of the study, with a single-subject design, was to measure correlations between self-reported rank ordering of a set of 23 soft skills and frontal gamma asymmetry emerging during exposure to the same set of soft skills stimuli, while simultaneously acquiring EEG data.

\section{Methods}

\section{Participants}

Fourteen individuals participated in the study over a 4-month period. The participants ranged in age from 29 to $67(M=47.8, S D=11.5)$, with 9 being male and 5 female. Ethnicity was primarily white; education included five each master's and bachelor's degrees; all were employed. See Table 1 for a breakout of age, gender, ethnicity, education, and occupation. All participants had normal or corrected-to-normal vision and no history of traumatic brain injury; there was no screening for substance and/or alcohol use/abuse. The external Institutional Review Board (IRB) of the Center for Applied Cognitive Research approved this study for the protection of human subjects in research, and all participants signed an informed consent document.

Table 1

Demographic Data

\begin{tabular}{cccccc}
\hline Case \# & Age & Gender & Ethnicity & Education & Occupation \\
\hline 1 & 67 & Male & White & Master's & Chief Executive Officer \\
2 & 48 & Male & White & Bachelor's & Facilitator/Trainer \\
3 & 48 & Male & White & Bachelor's & Fitness Trainer \\
4 & 47 & Male & White & High School & Consultant Supervisor \\
5 & 60 & Male & White & Master's & Consultant \\
6 & 29 & Female & White & Associate & Customer Support \\
7 & 59 & Female & White & Bachelor's & Consultant \\
8 & 62 & Male & White & Bachelor's & Executive Coach \\
9 & 37 & Female & Hispanic/Latino & Bachelor's & Sales Associates \\
10 & 41 & Male & White & Bachelor's & Lead Programmer/Analyst \\
11 & 56 & Male & White & Master's & Vice President \\
12 & 36 & Female & Middle Eastern & N/A & N/A \\
13 & 40 & Male & Hispanic/Latino & Master's & N/A \\
14 & 39 & Female & White & Master's & Executive Coach
\end{tabular}

Note. N/A = not available due to participant not providing that information.

\section{Materials}

To assess the extent to which participants have developed a set of 21 st century competencies, the TriMetrix® DNA, (Target Training International, Ltd;
Scottsdale, AZ) assessment was used to gain insights into the developmental level of these workrelated competencies. Table 2 provides a listing and definitions of each soft skill assessed. 


\section{Table 2}

Definitions of 23 Professional/Personal Soft Skill Competencies

\begin{tabular}{|c|c|}
\hline Analytical problem solving & Anticipating, analyzing, diagnosing, and resolving problems. \\
\hline Conflict management & Addressing and resolving conflict constructively. \\
\hline Continuous learning & $\begin{array}{l}\text { Taking initiative in learning and implementing new concepts, technologies, and/or } \\
\text { methods. }\end{array}$ \\
\hline Creativity/innovation & $\begin{array}{l}\text { Adapting traditional or developing new approaches, concepts, methods, models, } \\
\text { designs, processes, technologies, and/or systems. }\end{array}$ \\
\hline Customer service & Anticipating meeting and/or exceeding customer needs, wants, and expectations. \\
\hline Decision making & Utilizing effective processes to make decisions. \\
\hline Diplomacy & $\begin{array}{l}\text { Effectively handling difficult or sensitive issues by utilizing tact, diplomacy, and an } \\
\text { understanding of organizational culture, climate, and/or politics. }\end{array}$ \\
\hline Empathy & Identifying with and caring about others. \\
\hline Employee development/coaching & Facilitating and supporting the professional growth of others. \\
\hline Flexibility & Agility in adapting to change. \\
\hline Futuristic thinking & Imagining, envisioning, projecting, and/or predicting what has not yet been realized. \\
\hline Goal orientation & Energetically focusing efforts on meeting a goal, mission, or objective. \\
\hline Interpersonal skills & Effectively communicating, building rapport, and relating well to all kinds of people. \\
\hline Leadership & Achieving extraordinary business results through people. \\
\hline Management & $\begin{array}{l}\text { Achieving extraordinary results through effective management of resources, systems, } \\
\text { and processes. }\end{array}$ \\
\hline Negotiation & Facilitating agreements between two or more parties. \\
\hline Personal effectiveness & $\begin{array}{l}\text { Demonstrating initiative, self-confidence, resiliency, and a willingness to take } \\
\text { responsibility for personal actions. }\end{array}$ \\
\hline Persuasion & Convincing others to change the way they think, believe, or behave. \\
\hline Planning/organizing & Using logical, systematic, and orderly procedures to meet objectives. \\
\hline Presenting & Communicating effectively to groups. \\
\hline Self-management (time/priorities) & Demonstrating self-control and an ability to manage time and priorities. \\
\hline Teamwork & Working effectively and productively with others. \\
\hline Written communication & Writing clearly, succinctly, and understandably. \\
\hline
\end{tabular}

A crosswalk of these 23 skills against 21 st century soft skills is shown in Table 3 , as originally published in Gosselin, Cooper, Bonnstetter, and Bonnstetter (2013). Human resource personnel have found that an individual's hierarchy of competencies is key to their success and knowing what they are is essential to reaching their goals (National Research Council, 2012). This assessment is designed to assist in managing and developing a career. For many jobs, personal skills are as important as technical skills in producing superior performance. The TriMetrix $®$ DNA report describes what an individual "has done" in 23 research-based capacities related to the business environment.
Data from over 25,000 participants are used, on an annual basis, to validate total variance of the TriMetrix $®$ DNA, meaning that each of the 92 Likertscaled questions has a response range that encompasses the one through six choices from agree to disagree. For inter-rater reliability, a 360degree feedback survey is also used to assess the perception of others on an individual's evidencebased competencies; thus, triangulating between at least three auditors (e.g., peers, supervisors, subordinates, customers) to check perceptual agreement. 


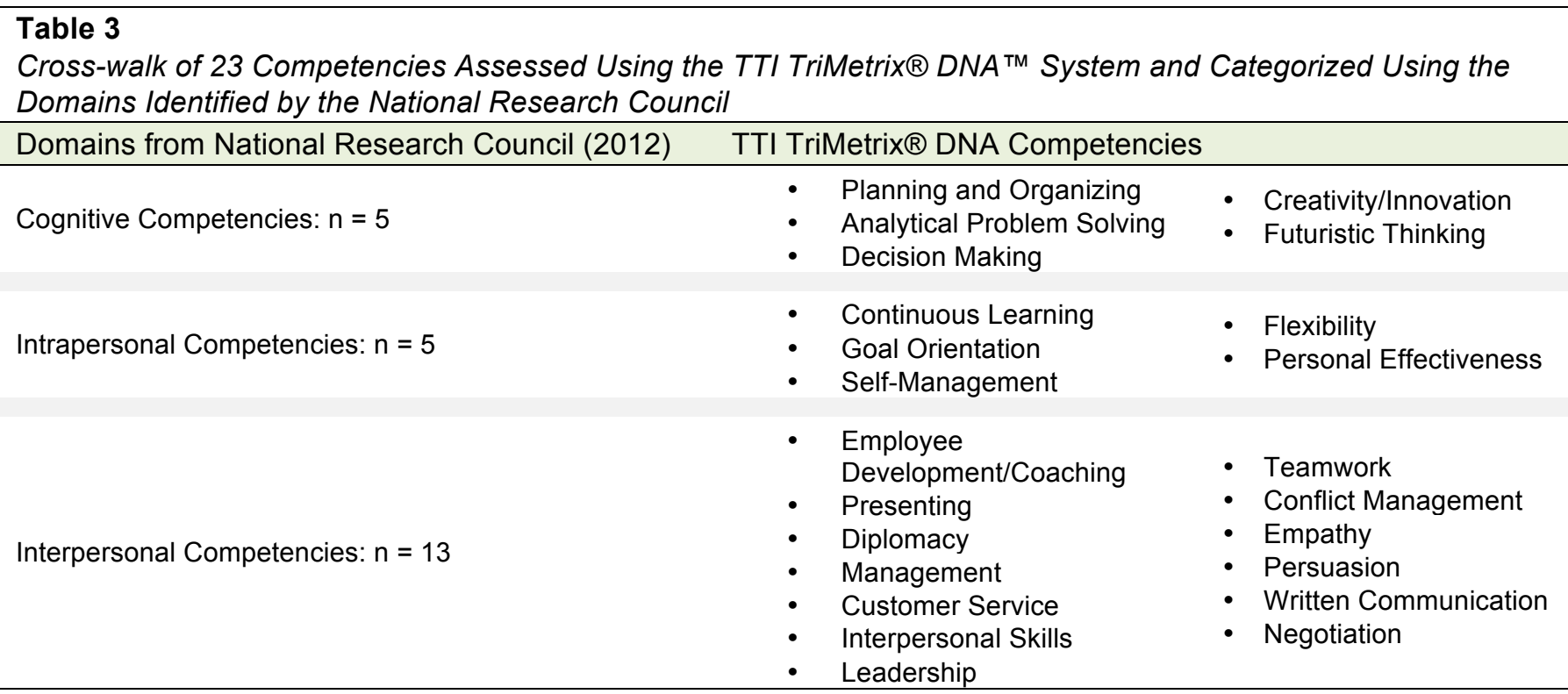

Note. Adapted from Gosselin et al. (2013).

\section{Procedures}

In the first phase of the procedure, each of the 14 participants completed the 30- to 40-min TriMetrix $®$ DNA assessment online, which included a set of questions concerning their personal perception of 23 competency accomplishment, as well as questions regarding others' perceptions of their abilities. This assessment was completed approximately two weeks prior to the EEG data collection phase. In this second phase, each participant was exposed to a randomized list of the same soft skills' key words while EEG was simultaneously acquired. Prior to commencement of EEG recording, subjects were told that the experiment was concerned with collecting their reaction to a set of words or short phrases that may or may not describe them. Once background and demographic data was collected participants were readied for EEG collection.

Each subject was fitted with an electrode cap (Electro-Cap International; Eaton, $\mathrm{OH}$ ) with 19 tin electrodes (plus a ground electrode), positioned to the International 10-20 system of electrode placement. The EEG amplifier was the Discovery 24E (Brainmaster Technologies; Bedford, $\mathrm{OH}$ ) with a sampling rate of 1,024 samples per second (data rate to the computer of 256 samples per second), an A/D conversion of 24-bit resolution, EEG bandwidth of $0.43-80 \mathrm{~Hz}$, and input impedance of 1,000 Gohm. EEG was acquired with the BrainAvatar software (Brainmaster Technologies; Bedford, $\mathrm{OH}$ ) with linked ears reference; electrode impedance was adjusted to be below $10 \mathrm{kohm}$. During the stimuli presentation, two auxiliary channels of the amplifier were used to record event start and stop markers. These markers were generated using a predesigned random set of soft skill stimuli built into an E-Prime 2.0 software (Psychology Software Tools, Inc.; Sharpsburg, PA). Prior to presenting the stimuli, 2 min of eyes-open and 2 min of eyes-closed EEG was collected for further analysis, if deemed necessary.

The procedure next involved presenting a series of screens shots positioned on a 20 -inch monitor, 1.5 $\mathrm{m}$ from the participant. They were told to watch the screen and focus attention on the words presented. The stimuli, presented in random order, appeared on the screen and remained for $1.5 \mathrm{~s}$, followed by a random blank screen from 1 to $5 \mathrm{~s}$. Further explanation of the basic setup and procedures used for data collection can be found in Bonnstetter, Collura, Hebets, and Bonnstetter (2012). This process allows for a series of modified event-related potential (ERP) type experiments. While not ERPs in the traditional sense, these events are still a timelocked stimulus to surface brain activity and measure transient electrical potential gamma shifts during cognitive processing. This patented process, referred to as Validating Ipsative Decision-making with Electroencephalography (VIDE; U.S. Patent No. $9,060,702,2015)$ provides the intensity of a person's emotional response to a stimulus, by measuring voxel activation and emotional directionality, by differentiating approach versus avoidance responses within the prefrontal cortex. 


\section{Data Analysis}

During post-processing analysis, averaged voxel values and SLORETA images were matched to the individual soft skill stimuli presented, to examine any associations. The region of interest (ROI) for analysis was identified as "frontal lobe," as predefined in the BrainAvatar imaging software (as designated by the Key Institute sLORETA model) and included only the left and right frontal lobes. In this software, the frontal lobe ROI is a very large area containing 2,176 voxels, which encompasses $35 \%(2,176$ of 6,239$)$ of all voxels.

Included in this ROI are the right and left Brodmann areas as follows: All of BA 6, 8, 9, 11, 44, 46; a majority or most of BA 4, 5, 10, 25, 45, 47; and a relative few voxels from $3,13,31,32,34,43$. In essence, this constitutes the first 2,176 voxels from The Key Institute sLORETA voxel index (http://www.uzh.ch/keyinst/loreta.htm). Table 4 provides a breakout, sorted by voxel number, of the specific voxels included in each BA making up the sLORETA frontal lobe ROI.

The resulting quantitative values were the average of the ROI voxels for the right and left frontal lobes. A measure of acceptance versus avoidance was calculated in the form of a numeric difference, termed the Approach-Avoidance-Differential (AAD), indicating the relative amount of energy in the right frontal lobe ROI, compared to the left. The AAD calculation is the average of the right hemisphere $R O I$ voxels minus the average of the left hemisphere ROI voxels $(1,088$ voxels per hemisphere). A negative value indicates greater left hemisphere activation and implies approach (i.e., a sense of accepting thoughts, feelings, and behavior) towards the stimulus word, a positive value indicates greater right hemisphere activation and implies avoidance (i.e., a sense of aversion) against the stimulus word, and a value near zero implies a neutral response.

\section{Table 4}

Brodmann Area Voxels Included in sLORETA Frontal Lobe ROI

\begin{tabular}{ccccc}
\hline $\begin{array}{c}\text { Brodmann } \\
\text { Area }\end{array}$ & $\begin{array}{c}\text { Total Number of } \\
\text { Voxels in BA }\end{array}$ & $\begin{array}{c}\text { Voxel Number } \\
\text { Range Included }\end{array}$ & $\begin{array}{c}\text { Number of Voxels } \\
\text { Included in BA }\end{array}$ & $\begin{array}{c}\text { Percentage } \\
\text { BA Included }\end{array}$ \\
\hline BA-10 & 272 & $1-268$ & 268 & $99 \%$ \\
BA-11 & 239 & $269-507$ & 239 & $100 \%$ \\
BA-13 & 248 & $508-517$ & 10 & $4 \%$ \\
BA-25 & 45 & $518-550$ & 33 & $73 \%$ \\
BA-3 & 129 & $551-553$ & 3 & $2 \%$ \\
BA-31 & 194 & $554-574$ & 21 & $11 \%$ \\
BA-32 & 155 & $575-596$ & 22 & $14 \%$ \\
BA-34 & 33 & $597-604$ & 8 & $24 \%$ \\
BA-4 & 146 & $605-743$ & 139 & $95 \%$ \\
BA-43 & 26 & $744-753$ & 10 & $38 \%$ \\
BA-44 & 56 & $754-809$ & 56 & $100 \%$ \\
BA-45 & 58 & $810-866$ & 57 & $98 \%$ \\
BA-46 & 46 & $867-912$ & 46 & $100 \%$ \\
BA-47 & 217 & $913-1125$ & 213 & $98 \%$ \\
BA-5 & 90 & $1126-1179$ & 54 & $60 \%$ \\
BA-6 & 554 & $1180-1733$ & 554 & $100 \%$ \\
BA-8 & 174 & $1734-1907$ & 174 & $100 \%$ \\
BA-9 & 269 & $1908-2176$ & 269 & $100 \%$ \\
\hline
\end{tabular}

Note. Total number of voxels in frontal lobe $\mathrm{ROI}=2,176$. 
Correlations were calculated for the paired occurrences between the self-reported ranking of the soft skills and the $A A D$ for the gamma asymmetry in response to the presented stimulus, for each participant. Given the ranked items are an ordinal variable, the Spearman's Rank correlation $\left(r_{s}\right)$ was calculated. This statistic does not evaluate linear relationships, but rather the strength of monotonic relationships (i.e., variables which change together in the same direction, but not necessarily at a constant rate). Therefore the $r_{s}$ results in a measure of directionality as well as the strength of the relationship. It is important to note, however, that the $r_{s}$ does not provide a predictive measure of linearity; therefore regression lines are not drawn on the data graphs in Figures 1 and 2 .

As a qualitative method of analysis, using sLORETA imaging, together with the VIDE process, a visual examination was made of the asymmetry in the frontal cortex, identifying gamma (38 to $42 \mathrm{~Hz}$ ) bursts to assess the underlying precognitive decisions behind the self-reported responses, at the moment of decision making (Collura, Zalaquett, et al., 2014). This process theoretically provides evidence that an evoked, emotionally laden response results in corresponding brain activity and exposes the match to TriMetrix $®$ DNA assessmentreported findings. This imaging process documents both the intensity of human emotional response as well as the directionality of the response. The process can be used, as in this study, for examining mental processes; but also has the potential for immediate open dialog with a client concerning issues that may become evident from this real-time mental imaging, as described in Collura, Bonnstetter, et al. (2014) as well as Collura, Zalaquett, et al. (2014).

\section{Results}

As shown in Table 5, when examining the top and bottom stimuli for all 14 individuals, we discovered a relationship between an individual's approach versus avoidance to a stimulus, based on the AAD and an examination of the sLORETA imaging. When the 14 participants' highest ranked personal skills are compared against their EEG responses to those same stimuli, there is a trending toward approach for their top five skills. This was indicated by a negative AAD score, together with an increased activation in their left frontal lobe, when examining gamma asymmetry in their frontal cortex.
Similarly, when we compared all 14 participants' five lowest ranked personal skills against their EEG responses to those same stimuli, we found an overall decrease in both their left and right frontal lobes, and AADs trending towards zero, indicating a neutral or reduced response, as if they did not recognize the stimuli/skill as being significant.

The rest of the 13 personal skills, those grouped between the top and bottom, seemed to shift or switch between approach, avoidance, or a neutral response. The response may depend on each individual's personal level of development, with a trend supporting the participants' likelihood of being emotionally more removed or disconnected from a skill, when they were moderately developed, or not developed at all in it. (The terms moderately developed and not developed come directly from the TriMetrix ${ }^{\circledR}$ DNA and are based on assessment population norms and the application of standard deviations.)

The resulting Spearman Rank correlations between the AAD and the soft skill rankings are shown in Table 6; for all cases $n=23$ with $d f=21$. Four cases $(4,5,6,8)$ yielded, in essence, no correlations with $r_{s}$ values ranging from -0.05 to 0.11 . Two cases $(1,7)$ resulted in low correlations with $r_{s}$ values of 0.29 and -0.28 , respectively. Two cases $(2,3)$ were moderately correlated with $r_{s}$ values of 0.52 and $p=.011$ for both. Four cases $(10,12,13$, 14) yielded strong correlations with $r_{s}$ values of $-0.69,0.73,-0.69,0.65$, respectively and $p$ values ranging from .000 to .001 . Two cases $(9,11)$ were nearly perfect correlations with $r_{s}$ values of 0.99 and 0.92 , respectively and $p=.000$ for both. Overall, $71 \%$ (10 of 14 ) of the cases resulted in monotonic correlations, indicating soft skill directionality; moreover, in many of these cases, a degree of intensity reduction can be seen as they move from their top skills to their bottom. Figures 1 and 2 provide a graphical representation of each participant's $A A D$ in response to the 23 stimuli; Figure 1 includes the 10 cases that resulted in correlations, while Figure 2 includes the four cases for which there were no correlations. 
Table 5

Approach-Avoidance Differential (AAD) per Soft skill Ranked Order per Participant

\begin{tabular}{|c|c|c|c|c|c|c|c|c|c|c|c|c|c|c|}
\hline \multirow{2}{*}{$\begin{array}{c}\text { Ranked } \\
\text { Order }\end{array}$} & \multicolumn{14}{|c|}{ Participant Number } \\
\hline & 1 & 2 & 3 & 4 & 5 & 6 & 7 & 8 & 9 & 10 & 11 & 12 & 13 & 14 \\
\hline $1 \mathrm{st}$ & -0.91 & -1.81 & -0.04 & 1.05 & 0.33 & 0.02 & 0.48 & -0.19 & -0.91 & 0.72 & -1.63 & -0.47 & 0.61 & -1.10 \\
\hline 2nd & -0.89 & -1.59 & -0.10 & 0.41 & -0.07 & -0.03 & -0.75 & 0.26 & -0.83 & 0.59 & -1.42 & -0.43 & 0.41 & -0.57 \\
\hline $3 r d$ & -0.86 & 0.90 & -0.02 & 0.30 & -0.27 & 0.52 & -0.04 & 0.51 & -0.81 & 0.44 & -1.25 & -0.40 & 0.22 & -0.30 \\
\hline 4th & -0.40 & -1.52 & -0.10 & 0.22 & -0.04 & 0.35 & -0.16 & 0.17 & -0.72 & 0.89 & -1.61 & -0.42 & 0.78 & -0.55 \\
\hline 5th & -0.37 & -1.14 & -0.04 & 0.38 & -0.34 & 0.21 & -0.50 & 0.04 & -0.59 & 0.63 & -1.22 & -0.39 & 0.52 & -0.55 \\
\hline 6th & 0.21 & -1.75 & -0.01 & 0.80 & 0.11 & -0.25 & -0.33 & 0.10 & -0.59 & 0.69 & -1.28 & -0.32 & 0.58 & -0.49 \\
\hline 7th & -0.39 & -0.55 & 0.11 & 0.62 & 0.14 & 0.47 & 0.16 & 0.21 & -0.57 & 0.78 & -1.35 & -0.30 & 0.67 & -0.36 \\
\hline 8th & 0.66 & -0.53 & 0.23 & 0.78 & -0.05 & -0.08 & 0.42 & -0.07 & -0.56 & 0.49 & -1.05 & -0.29 & 0.38 & -0.63 \\
\hline 9th & 0.58 & -0.80 & 0.39 & 1.00 & 0.11 & 0.15 & -0.49 & 0.55 & -0.53 & 1.08 & -1.61 & -0.27 & 0.97 & 0.02 \\
\hline 10th & 0.47 & -0.59 & 0.29 & 1.09 & 0.13 & 0.14 & -1.30 & -0.03 & -0.49 & 0.46 & -0.95 & -0.35 & 0.35 & -0.52 \\
\hline 11 th & 0.32 & -0.51 & 1.01 & 1.07 & -0.33 & 0.28 & -1.08 & -0.03 & -0.49 & 0.46 & -0.95 & -0.31 & 0.35 & -0.52 \\
\hline 12 th & 0.17 & -0.63 & 0.29 & 1.36 & 0.16 & -0.15 & 0.00 & 0.20 & -0.46 & 0.66 & -1.12 & -0.28 & 0.55 & -0.26 \\
\hline 13th & 0.19 & -1.03 & 0.44 & 1.11 & -0.09 & 0.22 & 0.48 & 0.05 & -0.45 & 0.50 & -0.95 & -0.25 & 0.39 & -0.40 \\
\hline 14th & 0.31 & -0.84 & 0.39 & 1.74 & -0.04 & 0.64 & -0.24 & 0.01 & -0.42 & 0.43 & -0.85 & -0.43 & 0.32 & -0.41 \\
\hline 15th & 0.27 & -0.80 & 0.56 & 1.21 & -0.14 & 0.12 & -0.56 & 0.34 & -0.42 & 0.76 & -1.18 & -0.36 & 0.65 & -0.08 \\
\hline 16th & 0.11 & -0.47 & 0.52 & 1.16 & 0.41 & 0.15 & 0.12 & 0.02 & -0.41 & 0.43 & -0.84 & -0.33 & 0.32 & -0.39 \\
\hline 17th & 0.09 & -0.83 & 0.26 & 1.27 & 0.29 & 0.14 & -0.38 & 0.08 & -0.40 & 0.48 & -0.88 & -0.28 & 0.37 & -0.32 \\
\hline 18th & 0.10 & -0.50 & 0.65 & 0.56 & -0.11 & 0.07 & -0.69 & 0.00 & -0.39 & 0.39 & -0.78 & -0.20 & 0.28 & -0.39 \\
\hline 19th & 0.07 & -0.67 & 0.05 & 0.69 & 0.25 & 0.17 & -0.59 & 0.22 & -0.27 & 0.49 & -0.37 & -0.26 & 0.19 & -0.05 \\
\hline 20th & 0.19 & -0.45 & 0.15 & 0.55 & 0.02 & 0.27 & -1.12 & 0.03 & -0.36 & 0.39 & -0.75 & -0.21 & 0.28 & -0.33 \\
\hline $21 \mathrm{st}$ & 0.21 & -0.71 & 0.14 & 0.24 & 0.08 & 0.16 & -0.24 & 0.14 & -0.18 & 0.39 & -0.57 & -0.20 & 0.14 & -0.04 \\
\hline 22nd & 0.15 & -0.30 & 0.15 & 0.17 & -0.07 & 0.46 & -0.91 & 0.10 & -0.21 & 0.28 & -0.30 & -0.19 & 0.12 & -0.11 \\
\hline 23rd & 0.03 & -0.32 & 0.34 & 0.14 & 0.03 & -0.05 & -0.13 & 0.11 & -0.23 & 0.21 & -0.44 & -0.29 & 0.18 & -0.12 \\
\hline
\end{tabular}

Note. Items shaded in pink are the AADs for the five highest ranked personal skills; items shaded in blue are the AADs for the five lowest ranked personal skills. 
Table 6

Spearman Rank-Order Correlations Between AAD and Soft skills Rankings ( $n=23 ; d f=21$ )

\begin{tabular}{ccc}
\hline Case\# & $r_{s}$ & $p$ \\
1 & $\mathbf{0 . 2 9}$ & .174 \\
2 & $\mathbf{0 . 5 2}$ & .011 \\
3 & $\mathbf{0 . 5 2}$ & .011 \\
4 & -0.05 & .833 \\
5 & 0.11 & .623 \\
6 & 0.05 & .819 \\
7 & $\mathbf{- 0 . 2 8}$ & .199 \\
8 & -0.04 & .846 \\
9 & $\mathbf{0 . 9 9}$ & .000 \\
10 & $\mathbf{- 0 . 6 9}$ & .000 \\
11 & $\mathbf{0 . 9 2}$ & .000 \\
12 & $\mathbf{0 . 7 3}$ & .000 \\
13 & $\mathbf{0 . 6 9}$ & .000 \\
14 & $\mathbf{0 . 6 5}$ & .001 \\
\hline
\end{tabular}

Note. Bold indicates presence correlation (low $=0.28$ to nearly perfect 0.99 ).

In keeping with the single-subject design, with the 10 $(71 \%)$ cases provide in Figure 1 , it is important to note that each graph must be read independently because each individual enters the experience with a different brain activity baseline. For example, Figure 1, graph 1, is the same data and image set depicted in Table 7. This individual shows both a movement from the left frontal cortex to the right, as well as a reduction in gamma activity as they approach their 23rd choice of skill development.
Moreover, Collura, Zalaquett, et al. (2014) posit persons may have an idiosyncratic approach or avoidance bias, to which this study may lend credence. For example, graphs 9, 11, 12 and 14, depict individuals having left hemisphere dominate gamma activity, with all negative AADs, which may be indicative of an approach bias as a baseline; then, show a reduction in intensity as they move from soft skills mastered to their last choice. Conversely, graphs 10 and 13 depict individuals having right hemisphere dominate gamma activity, with all positive AADs, which may be indicative of an avoidance bias as a baseline; and again, show intensity reduction from their first ranked item to a more neutral response to their last soft skills rankings. Here, again as suggested by Collura, Zalaquett, et al. (2014), these individuals may hold an overall bias towards a negative worldview, finding it difficult to embrace or to have a positive attitude towards much of anything. Rock (2008) describes dramatic effects that a negative baseline bias can have on perceptions, problem solving, decision making, stress management, collaboration, and even motivation. An intense avoidance baseline has been tied to an overly vigilant amygdala that results in a person being more tuned into threats than rewards. Thus the threat response is easily triggered. In fact, Baumeister and Leary (1995) explain how an avoidance baseline generates far more arousal in the limbic system than approach responses, thus leading to more intense and longer lasting effects from these perceived threats. Therefore, while Figure 1 shows directionality and, in some cases, an intensity reduction shift when examining their soft skill ranked ordering, it is important to note that each participants has a different baseline and therefore must be viewed from different baseline perspectives. 


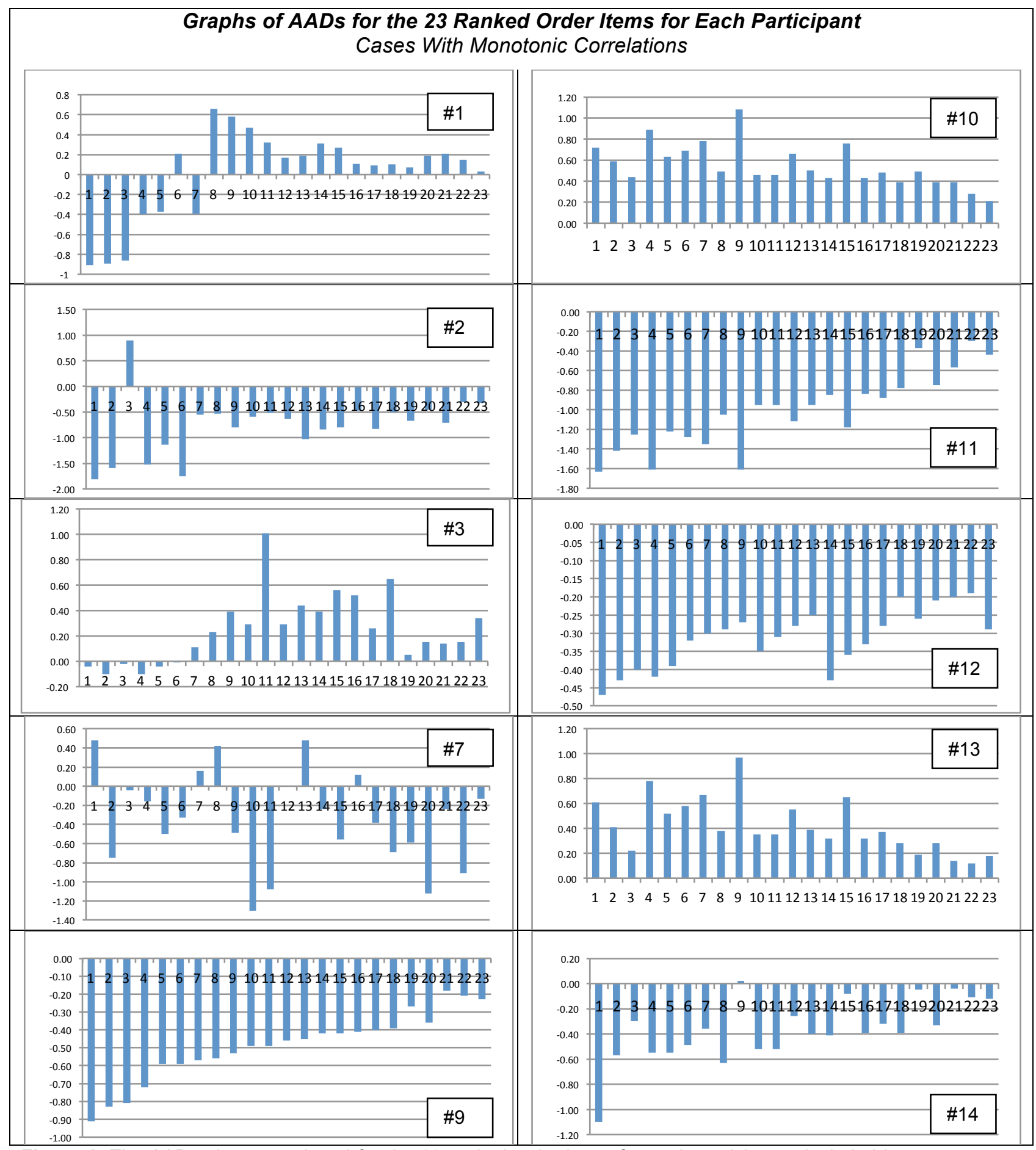

Figure 1. The AAD values are plotted for the 23 ranked order items for each participant. Included here are cases with monotonic correlations. 
Figure 2 depicts 4 participants (29\%) whose gamma asymmetry in response to the presented stimuli did not yield correlations with the soft skill ranking; thus, lacking directionality. As well, intensity reduction was not consistently seen. While data in these cases do show emotional expression, this expression does not occur in any predictable manner.

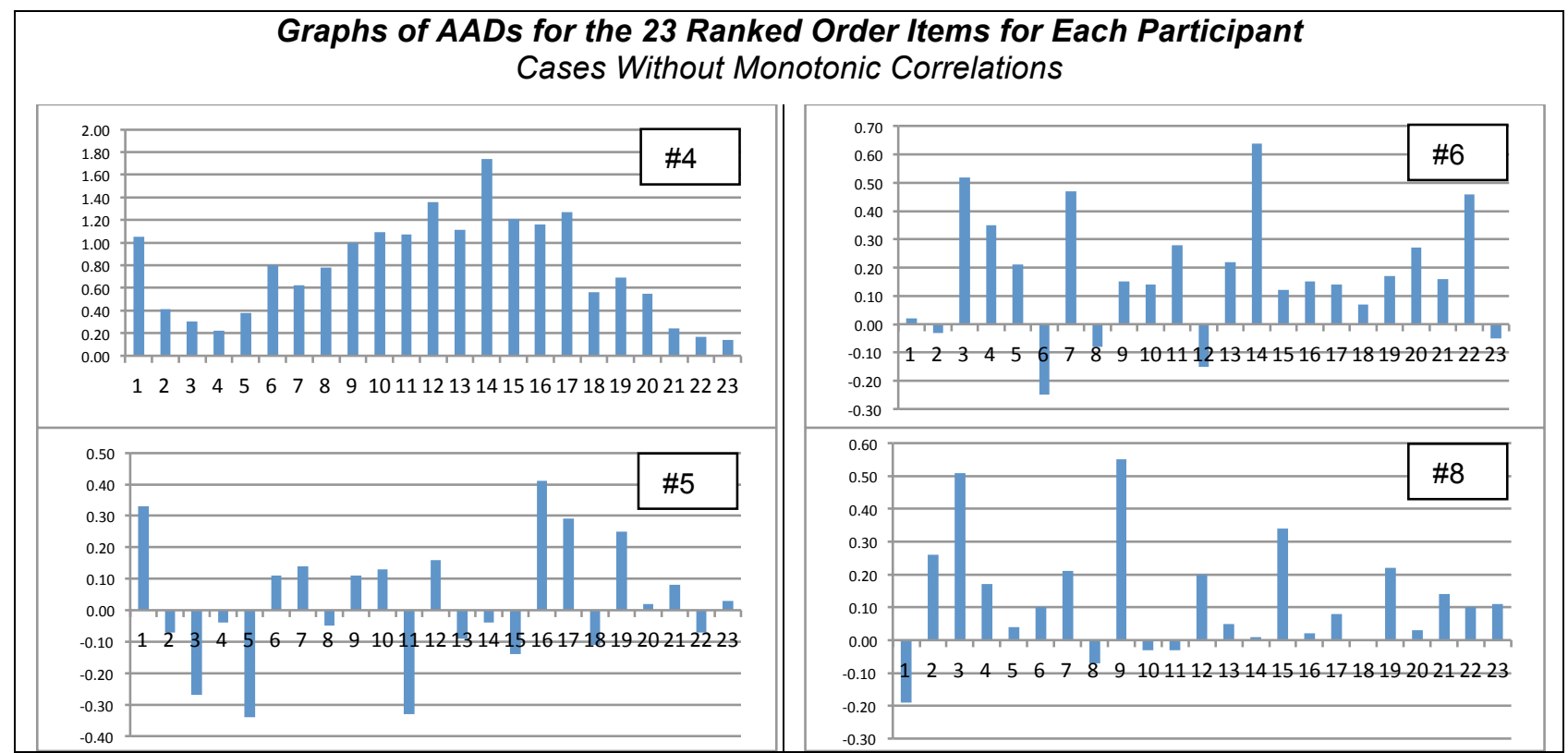

Figure 2. The $A A D$ values are plotted for the 23 ranked order items for each participant. Included here are cases without monotonic correlations.

\section{Single Case Illustrative Example}

As an illustrative, case study example, Table 7 summarizes the graphical (qualitative) and the AAD (quantitative) results for a single participant (\#1). This case also provides an example of the real-time voxel imaging available with this sLORETA implementation, using the VIDE process. The items are ranked in order of the individual's own self-rating of competence. Each item consists of the probe stimulus word (i.e., soft skill) presented, the AAD score, and the associated brain image responses shown as instantaneous activation patterns. In these images, the colors correspond to brain activity, gamma frequency; with red being the highest gamma activation and dark blue the lowest or no gamma activation. In this case, the AAD results are observed to rank in a manner associated with the individual ranking. In conjunction with these AADs, the brain images also show increased activity in the right side of the image (the client's left hemisphere) when $A A D$ values are negative (indicating approach or positive response), then moving toward more right-hemisphere activity when $A A D$ values are positive (indicating avoidance or negative response), as interest and self-assessment of competence decreases. Of particular note is the fact that the lowest ranked interest items seem to show an overall lack of response of any type, whether approach or avoidance, indicating an absence of response, not simply a negative response. 


\section{Table 7}

Frontal Gamma Asymmetry in Response to Soft Skills Stimuli in a Single Case (Participant \#1)

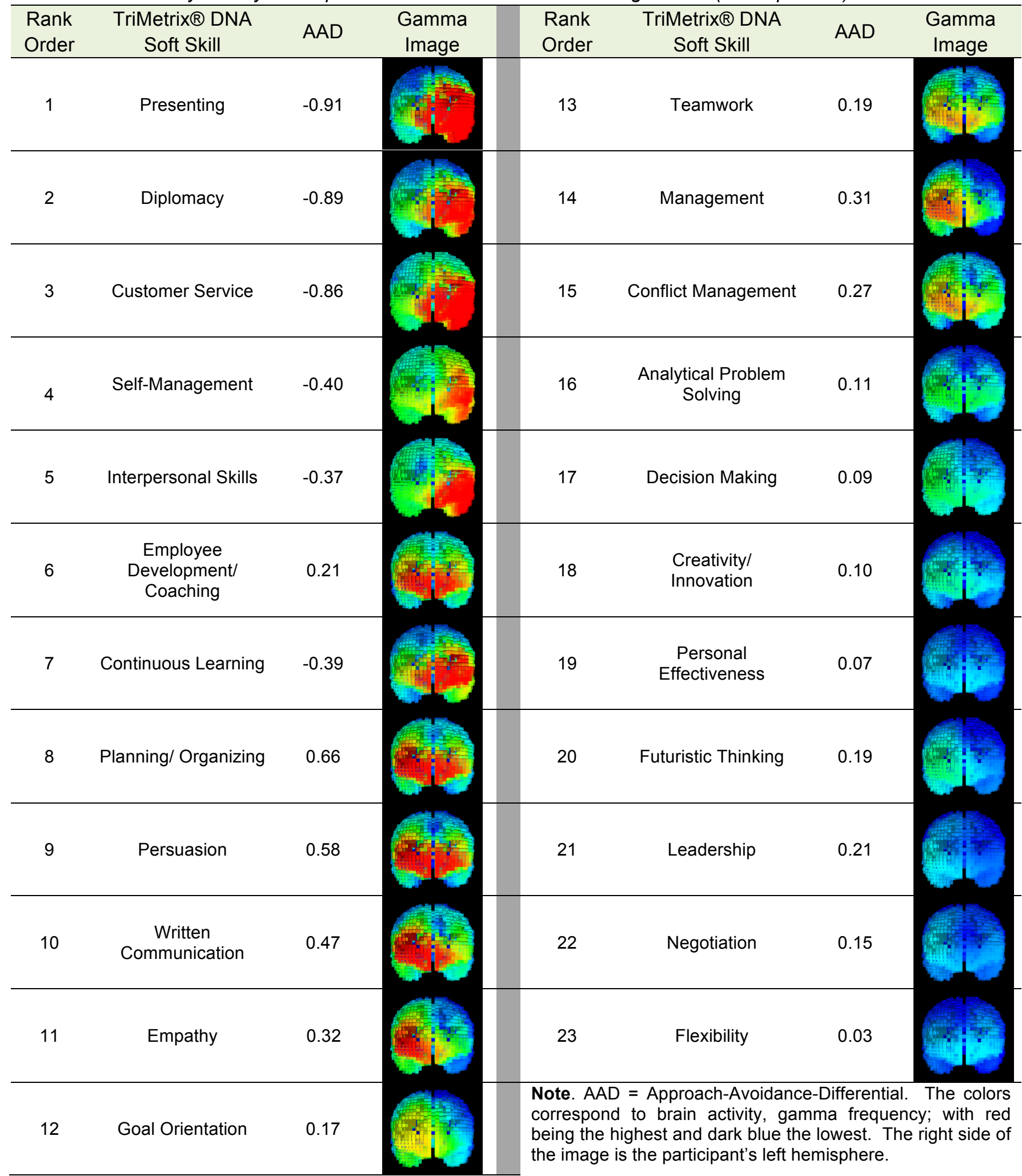




\section{Discussion}

The ability to match frontal gamma activity to selfreporting assessments would represent a new approach to self-report validation. To that end, the primary goal of this pilot study was to use sLORETA to measure the degree of association between selfreported rank ordering of a set of 23 soft skills and frontal gamma asymmetry during simultaneous exposure to the same set of soft skills. In this study, when individuals were asked to make choices leading to a rank ordering, or forced choice, the brain imaging and corresponding quantitative data yielded correlations in most cases $(71 \% ; 10$ of 14$)$. However, in four cases (29\%), while evidence of emotional expression was apparent, no correlations were found; thus no distinguishable pattern of directionality or intensity reduction emerged. Therefore, the degree that results of this pilot study can be generalized is limited. Yet, nonetheless, the use of SLORETA in this pilot study, together with real-time voxel imaging and the VIDE process, does present a novel approach to advancing this line of research in measuring gamma asymmetry with source localization EEG data. Further, in evaluating results on a case study basis, insights can be gained in how this data may be useful when interpreted on an individual level.

For example, in the case illustrated in Table 7, and in keeping with the model posited by Collura, Zalaquett, et al. (2014), while the individual appears to have a firm grasp on their top five soft skills, the brain precognitive responses to the remaining 17 skills may need to be discussed. For instance, it appears that the participant's precognitive position on planning/organizing, persuasion, written communication, empathy, and management tend toward avoidance. In other words, at a level outside of conscious awareness, this person may have deep beliefs or mindsets in place that would need to be exposed and altered before real progress toward new skill acquisition could occur. It might actually be easier to develop their worst rated skills (leadership, negotiation, and flexibility). At least these three skills appear to lack any previous emotional response and, therefore, may be easier to develop. Then, extending this perspective may also help to explain the lack of directionality and intensity reduction for the participants depicted in Figure 2. Within this context, a plausible theory may be that this group of individuals could have a poor sense of internal awareness such that it was difficult for them to provide accurate self-reports and/or rank orders of skills.
Limitations and Directions for Future Research While this study found beginning evidence of correlations between soft skill acquisitions (as defined by a self-reporting assessment) and gamma asymmetry, further refinement of the protocols are needed to build a reliable model. The time lag between the two data collection processes is one area to refine. Efforts need to be made to capture simultaneous data while filling out the self-report questionnaire and the corresponding EEG recordings. The 2-week window between events may have added uncontrolled variables. Moreover, the impact of the stimuli presentation order needs to be further addressed.

Then, comparison to some measure of selfawareness would be of benefit, as suggested as a hypothesis for Figure 2 participants. To investigate this, we intend to embark on cross-referencing participants with an additional instrument, to determine if other variables may adversely influence asymmetry. This triangulation will involve examining internal and external dimensional balance using an assessment designed to provide insights into external factors, including understanding of others, practical thinking, and system judgment, as well as internal factors including sense of self, role awareness, and self direction. Just as it is crucial to collect demographic and background histories, which may impact a study, we may have uncovered the need to document a person's ability to understand self in order to accurately assess precognitions.

Another variable that needs better control is the fact that within the population studied a wide range of behavioral styles and motivational factors were identified along with the soft skill data. Moreover, gender differences should be a future area of focus. Because of the limited number of participants, analysis of these subgroup factors was not possible. We may find that different behavioral styles react differently to soft skill acquisition. Thus, replication with larger sample sizes is needed.

Revisiting the research design in light of the limitations stated above represents the primary directions for future research. More so, further statistical validation of these methods is necessary for furthering this line of research. To take full advantage of the benefits of SLORETA source localization, isolating and investigating asymmetries in the most relevant BAs included in the frontal lobe $\mathrm{ROI}$, as well as other frequency bands, may prove advantageous. However, administering these protocols in real world contexts, such as during 
coaching sessions, job interviews, and possibly even in psychotherapeutic milieus (given proper ethical constraints), are promising areas for additional study, to evaluate the impact of potentially exposing hidden decision-making mechanisms of the preconscious mind.

\section{Conclusion}

The use of sLORETA real-time EEG data acquisition, and imaging, to investigate frontal gamma asymmetry in response to soft skill stimuli is innovative in its methodology as a new approach to the original work of Davidson, Pizzagalli, and other researchers. The correlation of self-reports' skill set by corresponding sLORETA brain imaging is encouraging; but more surprising, was the lack of brain response to those skills not possessed (i.e., the neutral responses). This may present beginning evidence of skills being experience-based and support a position that, in the lack of exposure to a skill, an individual may simply not have an emotional or memory connection to trigger a measurable brainactivation reaction. Nonetheless, it is important to remain mindful this pattern was found in $71 \%$ of participants; thus, concrete implications may be limited.

While much work remains, these results will inform further research, which has potential to lead to a means of validating survey results and at the same time exposing attempts at assessment manipulation. In spite of the exploratory nature of this study, advancing this line of research is warranted to investigate potential brain-based measures, which can objectively validate self-reported responses, and thereby provide meaningful indicators of emotional, cognitive, and motivational states, thus establishing advantageous tools in the field of organizational neuroscience.

\section{References}

Arthurs, O. J., \& Boniface, S. (2002). How well do we understand the neural origins of the fMRI BOLD signal? Trends in Neurosciences, 25(1), 27-31.

http://dx.doi.org/10.1016/S0166-2236(00)01995-0

Baumeister, R. F., \& Leary, M. R. (1995). The need to belong: Desire for interpersonal attachments as a fundamental human motivation. Psychological Bulletin, 117(3), 497-529. http://dx.doi.org/10.1037/0033-2909.117.3.497

Bedwell, W. L., Fiore, S. M., \& Salas, E. (January, 2011). Developing the 21st century (and beyond) workforce: A review of interpersonal skills and measurement strategies. Paper presented at the meeting of The National Academies, Board on Testing and Assessment and The National Academy of Education, Irvine, CA. http://www.eastbaycharterconnect.org/uploads/7/1/7/6/717622 0/developing_the_21st_century_and_beyond_workforce_a_review_of_interpersonal_skills__measurement_strategies _bedwell_et_al._2011.pdf
Bedwell, W. L., Fiore, S. M., \& Salas, E. (2014). Developing the future workforce: An approach for integrating interpersonal skills in the MBA classroom. Academy of Management Learning and Education, 13(2), 171-186. http://dx.doi.org/10.5465/amle.2011.0138

Bonnstetter, B. J., Bonnstetter, R. J., Hebets, D., \& Collura, T. F. (2015). U.S. Patent No. 9,060,702. Washington, DC: U.S. Patent and Trademark Office.

Bonnstetter, R. J., Collura, T. F., Hebets, D., \& Bonnstetter, B. J. (2012, Winter) Uncovering the belief behind the action. NeuroConnections, 20-23.

Collura, T. F, Bonnstetter, R. J., \& Zalaquett, C. (2014). Neurocounseling: Bridging Brain and Behavior. Seeing inside the client's mind. Counseling Today, 57(6), 24-27.

Collura, T. F., Zalaquett, C. P., Bonnstetter, R. J., \& Chatters, S. J. (2014). Toward an operational model of decision making, emotional regulation, and mental health impact. Advances in Mind-Body Medicine, 28(4), 18-33.

Davidson, R. J. (1992). Emotion and affective style: Hemispheric substrates. Psychological Science, 3(1), 39-43. http://dx.doi.org/10.1111/j.1467-9280.1992.tb00254.x

Davidson, R. J. (2000). Affective style, psychopathology, and resilience: Brain mechanisms and plasticity. American Psychologist, 55(11), 1196-1214. http://dx.doi.org/10.1037/0003-066X.55.11.1196

Davidson, R. J. (2002). Anxiety and affective style: Role of prefrontal cortex and amygdala. Biological Psychiatry, 51(1), 68-80. http://dx.doi.org/10.1016/S0006-3223(01)01328-2

Davidson, R. J. (2004). What does the prefrontal cortex "do" in affect: perspectives on frontal EEG asymmetry research. Biological Psychology, 67(1-2), 219-234. http://dx.doi.org/10.1016/j.biopsycho.2004.03.008

Davidson, R. J., Ekman, P., Saron, C. D., Senulis, J. A., \& Friesen, W. V. (1990). Approach-withdrawal and cerebral asymmetry: Emotional expression and brain physiology. Journal of Personality and Social Psychology, 58(2), 330341. http://dx.doi.org/10.1037/0022-3514.58.2.330

Davidson, R. J., Pizzagalli, D., Nitschke, J. B., \& Kalin, N. H. (2003). Parsing the subcomponents of emotion and disorders of emotion: Perspectives from affective neuroscience. In R. J. Davidson, H. H. Goldsmith, K. Scherer (Eds.), Handbook of Affective Sciences (pp. 8-24). New York: Oxford University Press.

Davidson, R. J., Schwartz, G. E., Saron, C., Bennett, J., \& Goleman, D. J. (1979). Frontal versus parietal EEG asymmetry during positive and negative affect. Psychophysiology, 16, 202-203.

Dweck, C. S. (2006). Mindset: The new psychology of success. New York, NY: Ballantine Books.

Gordon, E., Barnett, K. J., Cooper, N. J., Tran, N., \& Williams, L. M. (2008). An "Integrative Neuroscience" platform: Application to profiles of negativity and positivity bias. Journal of Integrative Neuroscience, 7(3), 345-366. http://dx.doi.org/10.1142/S0219635208001927

Gosselin, D., Cooper, S., Bonnstetter, R. J., \& Bonnstetter, B. J. (2013). Exploring the assessment of 21st century professional competencies of undergraduate students in environmental studies through a business-academic partnership. Journal of Environmental Studies and Sciences, 3(3), 359-368. http://dx.doi.org/10.1007/s13412-013-0140-1

Gosselin, D., Cooper, S., Bonnstetter, R. J, \& Bonnstetter, B. J. (2014). Erratum to: Exploring the assessment of 21st century professional competencies of undergraduate students in environmental studies through a business-academic partnership. Journal of Environmental Studies and Sciences, 4(2), 188-189. http://dx.doi.org/10.1007/s13412-014-0164-1

Harmon-Jones, E. (2004). Contributions from research on anger and cognitive dissonance to understanding the motivational functions of asymmetrical frontal brain activity. Biological Psychology, 67(1-2), 51-76. 
http://dx.doi.org/10.1016/j.biopsycho.2004.03.003

Hüsing, B., Jäncke, L., \& Tag, B. (2006). Impact Assessment Of Neuroimaging, Final report. Zürich: vdf Hochschulverlag.

Kanai, R., \& Rees, G. (2011). The structural basis of interindividual differences in human behaviour and cognition. Nature Reviews Neuroscience, 12(4), 231-242. http://dx.doi.org/10.1038/nrn3000

Lafferty, C. L., \& Alford, K. L. (2010). Neuroleadership: Sustaining research relevance into the 21 st century. SAM Advanced Management Journal, 75(3), 32-40.

McClelland, D. (1961). Human motivation. Cambridge, UK: Cambridge University Press.

Mitchell, J. P. (2008). Contributions of functional neuroimaging to the study of social cognition. Current Directions in Psychological Science, 17(2), 142-146. http://dx.doi.org/10.1111/j.1467-8721.2008.00564.x

Naccache, L., Gaillard, R., Adam, C., Hasboun, D., Clémenceau, S., Baulac, M., ... Cohen, L. (2005). A direct intracranial record of emotions evoked by subliminal words. Proceedings of the National Academy of Sciences of the United States of America, 102(21), 7713-7717. http://dx.doi.org/10.1073/pnas.0500542102

National Research Council. (2008). Research on future skill demands: A workshop summary. In M. L. Hilton \& Rapporteur (Eds.), Planning Committee on Research Evidence Related to Future Skill Demands. Center for Education. Division of Behavioral and Social Sciences and Education. Washington, DC: The National Academies Press.

National Research Council. (2012). Education for Life and Work: Developing Transferable Knowledge and Skills in the 21st Century. Committee on Defining Deeper Learning and 21st Century Skills. In J. W. Pellegrino \& M. L. Hilton, (Eds.), Board on Testing and Assessment and Board on Science Education, Division of Behavioral and Social Sciences and Education. Washington, DC: The National Academies Press.

Nitschke, J. B., Heller, W., Etienne, M. A., \& Miller, G. A. (2004). Prefrontal cortex activity differentiates processes affecting memory in depression. Biological Psychology, 67(1-2), 125143. http://dx.doi.org/10.1016/j.biopsycho.2004.03.004

Oakes, T. R., Pizzagalli, D. A., Hendrick, A. M., Horras, K. A., Larson, C. L., Abercrombie, H. C., ... Davidson, R. J. (2004). Functional coupling of simultaneous electrical and metabolic activity in the human brain. Human Brain Mapping, 21(4), 257-270. http://dx.doi.org/10.1002/hbm.20004

Pascual-Marqui, R. D. (2002). Standardized low-resolution brain electromagnetic tomography (sLORETA): Technical details. Methods and Findings in Experimental and Clinical Pharmacology, 24(Suppl. D), 5-12.

Pizzagalli, D. A., Greischar, L. L., \& Davidson, R. J. (2003). Spatio-temporal dynamics of brain mechanisms in aversive classical conditioning: high-density event-related potential and brain electrical tomography analyses. Neuropsychologia, 41(2), 184-194. http://dx.doi.org/10.1016/S0028-3932(02)00148-3

Pizzagalli, D. A., Nitschke, J. B., Oakes, T. R., Hendrick, A. M., Horras, K. A., Larson, C. L., ... Davidson, R. J. (2002). Brain electrical tomography in depression: the importance of symptom severity, anxiety, and melancholic features. Biological Psychiatry, 52(2), 73-85. http://dx.doi.org/10.1016/S0006-3223(02)01313-6

Pizzagalli, D., Pascual-Marqui, R. D., Nitschke, J. B., Oakes, T. R., Larson, C. L., Abercrombie, H. C., ... Davidson, R. J. (2001). Anterior cingulate activity as a predictor of degree of treatment response in major depression: Evidence from brain electrical tomography analysis. The American Journal of Psychiatry 158(3), 405-415. http://dx.doi.org/10.1176/appi.ajp.158.3.405

Rock, D. (2008). SCARF: a brain-based model for collaborating with and influencing others. NeuroLeadership, 1, 1-9.
Rolls, E. T. (1999). The Brain and Emotion. New York: Oxford University Press.

Tabibnia, G., Satpute, A. B., \& Lieberman, M. D. (2008). The sunny side of fairness: Preference for fairness activates reward circuitry (and disregarding unfairness activates selfcontrol circuitry). Psychological Science, 19(4), 339-347. http://dx.doi.org/10.1111/j.1467-9280.2008.02091.x

Thatcher, R. W. (2013). Latest developments in live z-score training: Symptom check list, phase reset, and LORETA $z$ score biofeedback. Journal of Neurotherapy, 17(1), 69-87. http://dx.doi.org/10.1080/10874208.2013.759032

The KEY Institute for Brain-Mind Research. (2014). Zürich, Switzerland. http://www.uzh.ch/keyinst/

Waldman, D. A., Balthazard, P. A., \& Peterson, S. J. (2011). Leadership and Neuroscience: Can We Revolutionize the Way That Inspirational Leaders Are Identified and Developed? Academy Of Management Perspectives, 25(1), 60-74. http://dx.doi.org/10.5465/AMP.2011.59198450

Yeager, D. S., \& Walton, G. M. (2011). Social-psychological interventions in education: They're not magic. Review of Educational Research, 81(2), 267-301. http://dx.doi.org/10.3102/0034654311405999

Received: May 9, 2015

Accepted: June 27, 2015

Published: July 15, 2015 